\title{
GERMINAÇÃO DE SEMENTES DE Adenanthera pavonina L. EM FUNÇÃO DE DIFERENTES TEMPERATURAS E SUBSTRATOS ${ }^{1}$
}

\author{
Ednaldo Bezerra de Souza ${ }^{2}$, Mauro Vasconcelos Pacheco ${ }^{3}$, Valderez Pontes Matos ${ }^{4}$ e Rinaldo Luiz \\ Caraciolo Ferreira ${ }^{5}$
}

\begin{abstract}
RESUMO - Este trabalho estudou a germinação das sementes de A. pavonina em diferentes substratos e regimes de temperatura. O delineamento experimental adotado foi inteiramente casualizado, em arranjo fatorial 6 x 5 (seis substratos: entre e sobre pó de coco, areia e vermiculita; e cinco temperaturas: 25, 30, 35, 20-30 e 20-35 ${ }^{\circ} \mathrm{C}$ ), com quatro repetições de 25 sementes cada. Os parâmetros germinação (\%), primeira contagem (\%), índice de velocidade de germinação, comprimento (cm/plântula) e massa seca (mg/plântula) da plântula foram avaliados. As temperaturas de $30 \mathrm{e} 35^{\circ} \mathrm{C}$ proporcionaram às sementes resultados satisfatórios de germinação, em todos os substratos testados. Os substratos pó de coco e areia permitiram bom desempenho germinativo, mostrando-se adequados para a avaliação da qualidade fisiológica de sementes de A. pavonina.
\end{abstract}

Palavras-chave: Sementes florestais, ecofisiologia da germinação e vigor.

\section{GERMINATION OF Adenanthera pavonina L. SEEDS AS A FUNCTION OF DIFFERENT TEMPERATURES AND SUBSTRATES}

\begin{abstract}
This work studied the germination of A. pavonina seeds in different conditions of temperature regime and substrate. The experiment was arranged in a $6 \times 5$ factorial, complete randomized design ( 6 substrates - sowing in and on: coconut fiber, sand and vermiculite; and 5 temperatures: 25, 30, 35, 20 30 and $20-35^{\circ} \mathrm{C}$ ), with four replications with 25 seeds each. The following parameters were analyzed: germination (\%), first germination count (\%), index of germination speed, length (cm/seedling) and dry matter weight ( $\mathrm{mg} / \mathrm{seedling).} \mathrm{The} \mathrm{best} \mathrm{results} \mathrm{of} \mathrm{germination} \mathrm{were} \mathrm{obtained} \mathrm{at} 30$ and $35^{\circ} \mathrm{C}$ in all substrates. Coconut fiber and sand substrates allowed satisfactory germinative performance, proving to be suitable for the evaluation of the physiological quality of A. pavonina seeds.
\end{abstract}

Keywords: Forest seeds, germination ecophysiology and vigor.

\section{INTRODUÇÃO}

Adenanthera pavonina L. (olho-de-pombo ou carolina), pertencente à família Leguminosae Mimosoideae (BARROSO et al., 1999), é uma espécie florestal originária da Ásia utilizada em reflorestamentos e como planta ornamental e forrageira (CORRÊA, 1978; AKKASAENG, 1989). No Brasil, foi introduzida há muitos anos e encontra-se bastante adaptada e largamente distribuída em todos os estados da Federação (CORRÊA, 1978).

Devida à grande diversidade de espécies nativas e exóticas de múltiplos usos, em enorme área territorial de vários aspectos edafoclimáticos, algumas plantas de uso na arborização urbana e na medicina popular - como A. pavonina-carecem de informações que possibilitem a avaliação fisiológica da qualidade das sementes.

\footnotetext{
${ }^{1}$ Recebido em $1^{\circ} .11 .2006$ e aceito para publicação em 13.03.2007.

${ }^{2}$ Autarquia de Ensino Superior de Arcoverde (Centro de Ensino Superior de Arcoverde) Rua: Gumercindo Cavalcanti, 420São Cristóvão, 56512-902 Arcoverde,PE. E-mail: <souzaednaldo@ hotmail.com>.

${ }^{3}$ Programa de Pós-Graduação em Ciência e Tecnologia de Sementes do Departamento de Fitotecnia da Universidade Federal de Pelotas. Caixa Postal 354, 96001-970 Pelotas,RS. E-mail: <pachecomv@ hotmail.com>.

${ }^{4}$ Departamento de Agronomia da Universidade Federal Rural de Pernambuco (UFPE), Av. D. Manoel de Medeiros, s/n, Dois Irmãos, 52171-900 Recife, PE. E-mail: <vpmatos@ig.com.br>.

${ }^{4}$ Departamento de Ciência Florestal da UFPE. E-mail: <rinaldof@ufrpe.br>.
} 
Para que as sementes germinem, elas devem dispor de condições internas e externas favoráveis (POPINIGIS, 1985). Assim, a capacidade máxima de germinação pode ser influenciada por fatores ambientais, como temperatura e substrato (BORGES e RENA, 1993).

A germinação constitui a fase do ciclo de vida que influencia diretamente a distribuição das plantas. Os estudos sobre a ecofisiologia da germinação e o conhecimento da biologia das sementes são importantes para que se compreendam todas as etapas de uma comunidade vegetal, bem como a sobrevivência e regeneração natural (BLACK e EL HADI, 1992; VÁZQUEZ-YANES e OROZCO-SEGOVIA, 1993).

Nesse contexto, a temperatura é responsável tanto por agir na velocidade de absorção de água como também em reações bioquímicas, influenciando a velocidade e uniformidade da germinação (BEWLEY e BLACK, 1994; CARVALHO e NAKAGAWA, 2000). Muitas sementes de essências florestais requerem regimes de temperatura específicos para poderem germinar, devido à forte influência das características ecológicas e do habitat onde se encontra cada espécie florestal. Assim, existem sementes que exigem temperaturas constantes, como Casearia sylvestris Sw. e Psychotria leiocarpa Cham. \& Schltdl. (ROSA e FERREIRA, 2001) ou alternadas, como foi observado em Sebastiania commersoniana (Baill.) Smith \& Downs (SANTOS e AGUIAR, 2000).

Outro importante fator ambiental a ser considerado é o substrato a ser utilizado no teste de germinação. As Regras para Análise de Sementes (RAS) (BRASIL, 1992) recomendam alguns substratos, como papel (toalha, filtro e mata-borrão), areia e solo. Entretanto, existem poucas recomendações para as espécies florestais, e outros tipos de substratos vêm sendo testados, a exemplo da vermiculita (ANDRADE et al., 2000) e do pó de coco (PACHECO et al., 2006).

Este estudo objetivou avaliar o comportamento germinativo das sementes de Adenanthera pavonina submetidas a diferentes temperaturas e substratos.

\section{MATERIAL E MÉTODOS}

As sementes de olho-de-pombo foram coletadas de cinco árvores localizadas na região metropolitana de Recife, Pernambuco, em dezembro de 2003. Em seguida, foram encaminhadas ao Laboratório de Sementes do Departamento de Agronomia da Universidade Federal
Rural de Pernambuco para beneficiamento e homogeneização. As sementes apresentavam teor de água de $10 \%$ por ocasião da instalação do experimento.

Os testes de germinação foram conduzidos em germinadores tipo Biochemical Oxigen Demand (B.O.D.), às temperaturas de $25,30,35,20-30$ e $20-35^{\circ} \mathrm{C}$ e fotoperíodo de $8 \mathrm{~h}$. Para as temperaturas alternadas, o período luminoso correspondeu à temperatura mais elevada.

Para superar a dormência tegumentar das sementes, estas foram escarificadas mecanicamente com lixa de ferro ( $n^{\circ}$ 50) na região oposta ao hilo (PANTOJA et al., 2006), desinfestadas com solução de hipoclorito de sódio 5\% durante 5 min e lavadas com água destilada. A semeadura foi realizada em caixas acrílicas transparentes de 11 x 11 x $3 \mathrm{~cm}$, com tampa. As sementes foram semeadas entre (camada de substrato com aproximadamente $1,0 \mathrm{~cm}$ de espessura acima da semente) e sobre os substratos pó de coco, areia e vermiculita, previamente autoclavados a $120^{\circ} \mathrm{C}$, durante $2 \mathrm{~h}$, e umedecidos com solução de Nistatina $0,2 \%$.

A areia é um substrato recomendado nas RAS (BRASIL, 1992), mas apresenta o inconveniente de drenar a água, acarretando ressecamento na parte superior do substrato, além de ser muito pesada, o que dificulta o manuseio das caixas plásticas no germinador (FIGLIOLIA et al., 1993).

Embora os substratos pó de coco e vermiculita não sejam mencionados nas Regras para Análise de Sementes, eles vêm sendo recentemente utilizados para a germinação de sementes de espécies florestais (MELO et al., 2005; PACHECO et al., 2006). Ambos os substratos são leves, de fácil manuseio, com boa capacidade de absorção de água, não exigem o reumedecimento diário e proporcionam bom desempenho germinativo das sementes.

O número de sementes germinadas foi avaliado diariamente, adotando-se como critério de germinação a emergência dos cotilédones e o surgimento do hipocótilo. Foram avaliados os seguintes parâmetros: germinação - a germinação foi avaliada a partir da porcentagem de plântulas normais (BRASIL, 1992) até o $13^{\circ}$ dia após a semeadura; primeira contagem - porcentagem de sementes germinadas até o 5ํ dia após o início do teste; índice de velocidade de germinação - determinado de acordo com a fórmula apresentada por Maguire (1962); comprimento de plântula - as plântulas normais de cada repetição 
foram medidas com o auxílio de uma régua graduada em milímetro; massa seca de plântula - as plântulas normais de cada repetição, após a retirada dos cotilédones, foram acondicionadas em sacos de papel, previamente identificadas, e levadas à estufa de ventilação forçada, regulada a $80^{\circ} \mathrm{C}$, durante $24 \mathrm{~h}$. Após esse período, as plântulas foram retiradas da estufa e pesadas em balança analítica, com precisão de $0,001 \mathrm{~g}$, sendo os resultados expressos em $\mathrm{mg} / \mathrm{plântula}$ (NAKAGAWA, 1999).

Os tratamentos foram distribuídos segundo o delineamento experimental inteiramente casualizado, e a análise de variância foi efetuada em arranjo fatorial $5 \times 6$ (cinco temperaturas e seis substratos), com quatro repetições de 25 sementes cada.

Para a análise dos dados, foi utilizado o software estatístico The SAS System for Windows (Statistical Analysis System), versão 8.02. SAS Institute Inc, 19992001, Cary, NC, USA. As médias foram comparadas pelo teste de Tukey a 5\% de probabilidade. Não houve necessidade de transformações dos dados, de acordo com os testes de normalidade e homogeneidade de variâncias realizados.

\section{RESULTADOS E DISCUSSÃO}

Verificou-se interação significativa $(p<0,05)$ entre substrato e temperatura em todos os parâmetros avaliados.

As combinações que proporcionaram maior porcentagem de germinação de sementes de olho-depombo (Quadro 1), realizada no décimo terceiro dia após a semeadura, foram obtidas quando se utilizaram as temperaturas constantes de 30 e $35^{\circ} \mathrm{C}$, independentemente do substrato testado. $\mathrm{Na}$ temperatura alternada de $20-30^{\circ} \mathrm{C}$, somente a combinação com os substratos sobre areia e sobre vermiculita proporcionou resultados satisfatórios de germinação. Ainda considerando a interação entre substrato e temperatura, na alternância de $20-35^{\circ} \mathrm{C}$ houve bom desempenho germinativo quando foram utilizados os substratos entre e sobre pó de coco, sobre areia e entre vermiculita, não apresentando diferença significativa entre essas combinações.

Quadro 1 - Valores médios de germinação (\%) e primeira contagem da germinação (\%) de sementes de Adenanthera pavonina L., submetidas a diferentes temperaturas e substratos

Table 1 - Germination (\%) and first germination count (\%) of Adenanthera pavonina L. seeds subjected to different temperatures and substrates

\begin{tabular}{|c|c|c|c|c|c|}
\hline \multirow[t]{2}{*}{ Substrato } & \multicolumn{5}{|c|}{ Temperatura $\left({ }^{\circ} \mathrm{C}\right)$} \\
\hline & 25 & 30 & 35 & $20-30$ & $20-35$ \\
\hline & \multicolumn{5}{|c|}{ Germinação (\%) } \\
\hline Entre pó de coco & $50 \mathrm{Bb}$ & $95 \mathrm{Aa}$ & 98 Aa & $29 \mathrm{Bc}$ & $91 \mathrm{ABa}$ \\
\hline Sobre pó de coco & $55 \mathrm{ABb}$ & $95 \mathrm{Aa}$ & $94 \mathrm{Aa}$ & $42 \mathrm{Bb}$ & $96 \mathrm{Aa}$ \\
\hline Entre areia & $68 \mathrm{Ab}$ & $98 \mathrm{Aa}$ & $96 \mathrm{Aa}$ & $77 \mathrm{Ab}$ & $78 \mathrm{Bb}$ \\
\hline Sobre areia & $49 \mathrm{BCb}$ & $82 \mathrm{Aa}$ & $95 \mathrm{Aa}$ & $86 \mathrm{Aa}$ & $92 \mathrm{ABa}$ \\
\hline Entre vermiculita & $46 \mathrm{BCc}$ & $91 \mathrm{Aa}$ & $96 \mathrm{Aa}$ & $74 \mathrm{Ab}$ & $90 \mathrm{ABa}$ \\
\hline$\underline{\text { Sobre vermiculita }}$ & $33 \mathrm{Cc}$ & $93 \mathrm{Aab}$ & $98 \mathrm{Aa}$ & $84 \mathrm{Aab}$ & $82 \mathrm{ABb}$ \\
\hline $\mathrm{CV}(\%)$ & 10,3 & & & & \\
\hline DMS (Substrato) & 7,4 & & & & \\
\hline \multirow[t]{2}{*}{ DMS (Temperatura) } & 6,5 & & & & \\
\hline & \multicolumn{5}{|c|}{ Primeira contagem da germinação (\%) } \\
\hline Entre pó de coco & $48 \mathrm{ABCb}$ & $90 \mathrm{Aa}$ & $63 \mathrm{Ab}$ & $0 \mathrm{Bc}$ & $0 \mathrm{Ac}$ \\
\hline Sobre pó de coco & $50 \mathrm{ABb}$ & $95 \mathrm{Aa}$ & $48 \mathrm{ABb}$ & $9 \mathrm{Bc}$ & $0 \mathrm{Ac}$ \\
\hline Entre areia & $64 \mathrm{Aab}$ & $82 \mathrm{Aa}$ & $49 \mathrm{ABb}$ & $7 \mathrm{Bc}$ & $0 \mathrm{Ac}$ \\
\hline Sobre areia & $46 \mathrm{ABCb}$ & $50 \mathrm{Bab}$ & $44 \mathrm{Bb}$ & $67 \mathrm{Aa}$ & $0 \mathrm{Ac}$ \\
\hline Entre vermiculita & $42 \mathrm{BCa}$ & $32 \mathrm{Ba}$ & $32 \mathrm{Ba}$ & $7 \mathrm{Bb}$ & $0 \mathrm{Ab}$ \\
\hline Sobre vermiculita & $30 \mathrm{Cb}$ & $84 \mathrm{Aa}$ & $0 \mathrm{Cc}$ & $17 \mathrm{Bbc}$ & $0 \mathrm{Ac}$ \\
\hline$\overline{\mathrm{CV}(\%)}$ & 26,2 & & & & \\
\hline DMS (Substrato) & 8,5 & & & & \\
\hline DMS (Temperatura) & 7,4 & & & & \\
\hline
\end{tabular}

Médias seguidas pela mesma letra maiúscula na coluna e minúscula na linha não diferem entre si, pelo teste de Tukey a 5\% de probabilidade. 
Em sementes de Caesalpiniae ferrea Mart. Ex Tul., a temperatura de $30^{\circ} \mathrm{C}$ também mostrou-se adequada para a otimização do desempenho germinativo, além de ter favorecido a redução do tempo médio de germinação (LIMA et al., 2006). Esses resultados reforçam a afirmação de que a maioria das espécies tropicais e subtropicais apresenta potencial germinativo máximo na faixa de temperatura entre 20 e $30^{\circ} \mathrm{C}$ (BORGES e RENA, 1993). Entretanto, os resultados deste estudo indicaram que as sementes de olho-de-pombo são capazes de extrapolar essa faixa, semelhantemente ao que também ocorre com outras espécies, como Dinizia excelsa Ducke (VARELA et al., 2005a), Acosmium nitens (Vog.) Yakovlev (VARELA et al., 2005b) e Schizolobium amazonicum Huber ex Ducke (RAMOS et al., 2006).

Com relação aos dados da primeira contagem da germinação (Quadro 1), realizada no quinto dia após a semeadura, os melhores resultados foram observados quando se utilizou a temperatura de $30^{\circ} \mathrm{C} \mathrm{em}$ todos os substratos, exceto sobre areia e entre vermiculita. Também foram obtidas boas combinações quando as sementes foram semeadas no substrato entre areia a $25^{\circ} \mathrm{C}$ e sobre areia a $20-30^{\circ} \mathrm{C}$. À $20-35^{\circ} \mathrm{C}$ não houve germinação na primeira contagem em nenhum dos substratos testados, indicando que a alternância/regime de temperatura retarda a velocidade das reações bioquímicas que regulam todo o processo metabólico da germinação.

Semelhantemente ao observado na porcentagem de germinação (Quadro 1), o Quadro 2 mostra que as temperaturas constantes de $30^{\circ} \mathrm{C} \mathrm{em}$ todos os substratos, exceto sobre areia e entre vermiculita, $35^{\circ} \mathrm{C}$ exceto sobre vermiculita e a temperatura alternada de $20-30^{\circ} \mathrm{C}$ no substrato sobre areia proporcionaram às sementes de A. pavonina os maiores valores da velocidade de germinação.

A temperatura ótima para a germinação de sementes está diretamente associada às características ecológicas da espécie (PROBERT, 1992). Nas temperaturas constantes, obtiveram-se porcentagem e velocidade maiores de germinação das sementes, em comparação com as temperaturas alternadas. A ocorrência de germinação em ambos os regimes de temperatura mostra a tendência que as sementes dessa espécie têm de germinar em pequenas clareiras, o que evidencia uma adaptação às flutuações térmicas naturais do ambiente. No entanto, parecem ser mais capazes de se adaptarem às condições de sub-bosque, nas quais predominam amplitudes térmicas menores.

Nesse contexto, as sementes de A. pavonina apresentam caráter estratégico de sobrevivência, pois são capazes de suportar as condições adversas do ambiente, germinar em ampla faixa de temperatura e garantir a perpetuação da espécie numa comunidade vegetal.

Os substratos e temperaturas utilizados neste estudo proporcionaram taxas de germinação elevadas. No entanto, além da porcentagem de germinação, o conceito de temperatura ótima deve considerar outros aspectos, como o vigor (LABOURIAU, 1983), de forma que seja possível proporcionar informações adicionais à interpretação dos resultados.

As melhores combinações para comprimento (Quadro 3) foram alcançadas quando as plântulas foram submetidas às temperaturas de $30^{\circ} \mathrm{C}$ nos substratos entre e sobre pó de coco e sobre vermiculita e a $35^{\circ} \mathrm{C}$ em todos os substratos, exceto entre areia. Na massa seca da plântula

Quadro 2 - Valores médios do índice de velocidade de germinação de sementes de Adenanthera pavonina L., submetidas a diferentes temperaturas e substratos

Table 2 - Germination speed index of Adenanthera pavonina L. seeds subjected to different temperatures and substrates

\begin{tabular}{lccccc}
\hline Substrato & \multicolumn{4}{c}{ Temperatura $\left({ }^{\circ} \mathrm{C}\right)$} \\
\hline Entre pó de coco & 25 & 30 & 35 & $20-30$ & $20-35$ \\
Entre pó de coco & $50 \mathrm{Bb}$ & $95 \mathrm{Aa}$ & $98 \mathrm{Aa}$ & $29 \mathrm{Bc}$ & $91 \mathrm{ABa}$ \\
Sobre pó de coco & $2,48 \mathrm{Bb}$ & $4,71 \mathrm{ABa}$ & $4,61 \mathrm{Aa}$ & $0,93 \mathrm{Cc}$ & $3,00 \mathrm{ABCb}$ \\
Entre areia & $2,70 \mathrm{ABb}$ & $4,75 \mathrm{ABa}$ & $4,20 \mathrm{Aa}$ & $1,52 \mathrm{Cc}$ & $3,40 \mathrm{Ab}$ \\
Sobre areia & $3,34 \mathrm{Ab}$ & $4,76 \mathrm{Aa}$ & $4,40 \mathrm{Aa}$ & $2,51 \mathrm{Bc}$ & $2,35 \mathrm{Cc}$ \\
Entre vermiculita & $2,40 \mathrm{BCc}$ & $3,72 \mathrm{Cab}$ & $4,10 \mathrm{Aa}$ & $3,76 \mathrm{Aab}$ & $3,20 \mathrm{ABb}$ \\
Sobre vermiculita & $2,24 \mathrm{BCc}$ & $3,96 \mathrm{BCa}$ & $4,17 \mathrm{Aa}$ & $2,40 \mathrm{Bbc}$ & $3,02 \mathrm{ABCb}$ \\
CV (\%) & $1,61 \mathrm{Cc}$ & $4,56 \mathrm{ABa}$ & $3,20 \mathrm{Bb}$ & $3,11 \mathrm{ABb}$ & $2,46 \mathrm{BCb}$ \\
DMS (Substrato) & 11,7 & & & \\
DMS (Temperatura) & 0,3 & & & \\
\hline
\end{tabular}

Médias seguidas pela mesma letra maiúscula na coluna e minúscula na linha não diferem entre si, pelo teste de Tukey a 5\% de probabilidade.

R. Árvore, Viçosa-MG, v.31, n.3, p.437-443, 2007 
(Quadro 3) houve maior interação entre substrato e temperatura (não apresentando diferença significativa), nas seguintes condições: a $30^{\circ} \mathrm{C}$, entre e sobre pó de coco; a $35^{\circ} \mathrm{C}$ em todos os substratos, exceto sobre pó de coco; a $20-30{ }^{\circ} \mathrm{C}$ com os substratos entre e sobre vermiculita; e a $20-35^{\circ} \mathrm{C}$ somente com o substrato sobre vermiculita. Nesse contexto, pode-se verificar que as plântulas de A. pavonina apresentaram maior crescimento e maior transferência de massa seca quando as sementes foram submetidas, principalmente, às temperaturas constantes mais elevadas, havendo poucas combinações satisfatórias nas interações entre as temperaturas alternadas com os substratos.

Na Europa e nos Estados Unidos da América, o pó de coco tem sido importado, principalmente do Sri Lanka, em larga escala para substituir a turfa na horticultura intensiva (VAVRINA et al., 1996). No Brasil, o pó de coco vem sendo recentemente utilizado como substrato para germinação de sementes de espécies florestais. Em sementes de Myracrodruon urundeuva Fr. All., esse substrato permitiu bom desempenho germinativo (atingindo $91 \%$ de germinação) e não exigiu reumedecimento diário, mostrando-se mais adequado à avaliação da qualidade fisiológica dessas sementes (PACHECO et al., 2006). A utilização do pó de coco como substrato também contribui para a redução da deposição dos resíduos sólidos nos aterros sanitários, além de se apresentar como um substituto para substratos como a areia e a vermiculita, os quais produzem impactos ambientais negativos (ROSA et al., 2001).

Em sementes de Mimosa regnelli Benthan, a areia foi recomendada para testes de germinação por ter proporcionado boas taxas germinativas, além de ser livre de microrganismos, de baixo custo e reutilizável (FOWLER e CARPANEZZI, 1997). Apesar de a areia ser um substrato pesado, o que dificulta o manuseio das caixas plásticas no germinador, e exigir reposição de água, mesmo assim ela permitiu boas combinações de germinação e vigor para as sementes de olho-de-pombo. Fanti e Perez (1999) também constataram que, ao utilizar areia para germinação de sementes de A. pavonina, houve dificuldade no que diz respeito à manutenção da umidade, uma vez que houve desuniformidade na capacidade de retenção e distribuição da água. A reposição da umidade deve-se ao fato de este substrato drenar a água, fazendo que a parte superior fique ressecada (FIGLIOLIA et al., 1993).

Quadro 3 - Comprimento (cm/plântula) e massa seca de plântulas (mg/plântula) de Adenanthera pavonina L. oriundas de sementes submetidas a diferentes temperaturas e substratos

Table 3 - Length ( $\mathrm{cm} / \mathrm{see}$ sling) and dry matter weight ( $\mathrm{mg} / \mathrm{seedling}$ ) of Adenanthera pavonina L. seedlings derived from seeds subjected to different temperatures and substrates

\begin{tabular}{|c|c|c|c|c|c|}
\hline \multirow[t]{2}{*}{ Substrato } & \multicolumn{5}{|c|}{ Temperatura $\left({ }^{\circ} \mathrm{C}\right)$} \\
\hline & 25 & 30 & 35 & $20-30$ & $20-35$ \\
\hline & \multicolumn{5}{|c|}{ Comprimento da plântula ( $\mathrm{cm} /$ plântula) } \\
\hline Entre pó de coco & $8,7 \mathrm{Ab}$ & $14,5 \mathrm{Aa}$ & $15,0 \mathrm{Aa}$ & $5,5 \mathrm{Ac}$ & $5,1 \mathrm{ABc}$ \\
\hline Sobre pó de coco & $8,0 \mathrm{Ab}$ & $14,1 \mathrm{Aa}$ & $14,3 \mathrm{ABa}$ & 4,4 Ac & $5,5 \mathrm{Ac}$ \\
\hline Entre areia & $5,6 \mathrm{Bb}$ & $9,4 \mathrm{Cb}$ & $12,7 \mathrm{Ba}$ & $6,0 \mathrm{Ac}$ & $4,4 \mathrm{ABc}$ \\
\hline Sobre areia & $3,4 \mathrm{Cb}$ & $4,8 \mathrm{Db}$ & $15,0 \mathrm{Aa}$ & $3,8 \mathrm{Ab}$ & $3,3 \mathrm{Bb}$ \\
\hline Entre vermiculita & $8,2 \mathrm{Ac}$ & $11,0 \mathrm{BCb}$ & 15,1 Aa & $5,5 \mathrm{Ad}$ & $5,0 \mathrm{ABd}$ \\
\hline Sobre vermiculita & $5,2 \mathrm{BCb}$ & $12,7 \mathrm{Aba}$ & $14,2 \mathrm{ABa}$ & $4,6 \mathrm{Ab}$ & $5,3 \mathrm{ABb}$ \\
\hline$\overline{\mathrm{CV}(\%)}$ & 12,5 & & & & \\
\hline DMS (Substrato) & 0,9 & & & & \\
\hline \multirow[t]{2}{*}{ DMS (Temperatura) } & 0,8 & & & & \\
\hline & \multicolumn{5}{|c|}{ Massa seca da plântula (mg/plântula) } \\
\hline Entre pó de coco & $21,0 \mathrm{Ad}$ & $50,2 \mathrm{ABa}$ & $46,3 \mathrm{Aab}$ & $35,0 \mathrm{ABbc}$ & 30,0 Acd \\
\hline Sobre pó de coco & $23,7 \mathrm{Ac}$ & $63,0 \mathrm{Aa}$ & $41,4 \mathrm{Ab}$ & $22,0 \mathrm{Bc}$ & $34,1 \mathrm{Abc}$ \\
\hline Entre areia & $16,1 \mathrm{Ac}$ & $44,0 \mathrm{Bab}$ & $49,2 \mathrm{Aa}$ & $23,1 \mathrm{Bc}$ & $36,2 \mathrm{Ab}$ \\
\hline Sobre areia & $17,3 \mathrm{Abc}$ & $13,2 \mathrm{Dc}$ & $48,0 \mathrm{Aa}$ & $26,5 \mathrm{ABb}$ & $27,8 \mathrm{Ab}$ \\
\hline Entre vermiculita & $14,4 \mathrm{Ac}$ & $30,1 \mathrm{Cb}$ & $50,0 \mathrm{Aa}$ & $37,7 \mathrm{Aab}$ & $34,6 \mathrm{Ab}$ \\
\hline Sobre vermiculita & $17,0 \mathrm{Ab}$ & $29,1 \mathrm{Cab}$ & $40,3 \mathrm{Aa}$ & $28,1 \mathrm{ABab}$ & $38,2 \mathrm{Aa}$ \\
\hline$\overline{\mathrm{CV}(\%)}$ & 20,4 & & & & \\
\hline DMS (Substrato) & 4,1 & & & & \\
\hline DMS (Temperatura) & 3,6 & & & & \\
\hline
\end{tabular}

Médias seguidas pela mesma letra maiúscula na coluna e minúscula na linha não diferem entre si, pelo teste de Tukey a 5\% de probabilidade. 
Embora a vermiculita apresente boa capacidade de absorção de água, a umidade era perdida com maior rapidez, tornando necessário o reumedecimento freqüiente desse substrato, principalmente em temperaturas mais elevadas. Resultados semelhantes também foram observados em sementes de Momordica charantia L., pois a vermiculita não proporcionou resultados satisfatórios, reduzindo a porcentagem e a velocidade de germinação (BEZERRA et al., 2002). Consta nas Regras para Análise de Sementes (BRASIL, 1992) que se deve evitar, sempre que possível, o reumedecimento dos substratos após a semeadura, uma vez que esse procedimento pode causar variações adicionais aos resultados.

Assim, pôde-se verificar que há grande variação no que diz respeito ao desempenho germinativo das sementes em relação aos substratos e temperaturas utilizados em condições de laboratório, tornando necessária a definição do ambiente que melhor proporcione a expressão máxima do vigor de cada espécie florestal.

\section{CONCLUSÕES}

- Considerando os testes de germinação e de vigor, verificou-se que as temperaturas de 30 e $35^{\circ} \mathrm{C}$ proporcionam às sementes de Adenanthera pavonina L. bom desempenho germinativo em todos os substratos testados.

- Os substratos areia e pó de coco permitem boa capacidade germinativa, mostrando-se mais adequados à avaliação da qualidade fisiológica de sementes de A. pavonina.

\section{REFERÊNCIAS}

AKKASAENG, R. Evaluation of trees and shrubs for forage and fuelwood in Northeast Thailand. International Tree Crops Journal, v.5, n.4, p.209-220, 1989.

ANDRADE, A. C. S. et al. desenvolvimento pósseminal. Pesquisa Agropecuária

Brasileira, v.35, n.3, p.609-615, 2000.

BARROSO, G. M. et al. Frutos e sementes: morfologia aplicada à sistemática de dicotiledôneas. Viçosa, MG: Universidade Federal de Viçosa, 1999. 443p.

R. Árvore, Viçosa-MG, v.31, n.3, p.437-443, 2007
BEWLEY, J. D.; BLACK, M. Seeds: physiology of development and germination. New York: Plenum Press, 1994. 445p.

BEZERRA, A. M. E. et. al. Germinação e desenvolvimento de plântulas de melão-de-sãocaetano em diferentes ambientes e substratos. Ciência Agronômica, v.33, n.1, p.39-44, 2002.

BLACK, R.; EL HADI, F. M. Presouring treatments of Acacia senegal seed: germination and growth. Tropical Agricultural, v.69, p.15-20, 1992.

BORGES, E. E. L.; RENA, A. B. Germinação de sementes. In: AGUIAR, I.B.; PINÃ-RODRIGUES, F. C. M.; FIGLIOLIA, M. B. Sementes florestais tropicais. Brasília: ABRATES, 1993. p.83-135.

BRASIL. Ministério da Agricultura e Reforma Agrária. Regras para análise de sementes. Brasília: SNDA/DNDV/CLAV, 1992.365p.

CARVAlho, N. M.; NAKAGAWA, J. Sementes: ciência, tecnologia e produção. Jaboticabal: FUNEP, 2000. 588p.

CORRÊA, M. P. Dicionário das plantas úteis do Brasil e das exóticas cultivadas. Rio de Janeiro: Imprensa Nacional, 1978. v.2. p.70.

FANTI, S. C.; PEREZ, S. C. J. G. A. Influência do substrato e do envelhecimento acelerado na germinação de olho-de-dragão (Adenanthera pavonina L. - Fabaceae). Revista Brasileira de Sementes, v.21, n.2, p.135-141, 1999.

FIGLIOLIA, M. B.; OLIVEIRA, E. C.; PINÃRODRIGUES, F. C. M. Análise de sementes. In: AGUIAR, I. B.; PINÃ-RODRIGUES, F. C. M.; FIGLIOLIA, M. B. Sementes florestais tropicais. Brasília: ABRATES, 1993. p.137-174.

FOWLER, J. A. P.; CARPANEZZI, A. A.

Influência do tipo de substratos e de temperaturas na germinação de sementes de juquiri (Mimosa regnelii Benthan). Colombo: Embrapa-CNPF, 1997. p.1-2. (Embrapa-CNPF: Comunicado Técnico, 16).

LABOURIAU, L.G. A germinação das sementes. Washington: Secretaria-Geral da Organização dos Estados Americanos, 1983. 174p. 
LIMA, J. D. et al. Efeito da temperatura e do substrato na germinação de sementes de Caesalpiniae ferrea Mart. ex Tul. (Leguminosae, Caesalpinioidae). Revista Árvore, v.30, n.4, p.513-518, 2006.

MAGUIRE, J. D. Speed of germination-aid in selection and evaluation for seedlings emergence and vigor. Crop Science, v.2, n.1, p.176-177, 1962.

MELO, R. R.; FERREIRA, A. G.; JUNIOR, F. R. Efeito de diferentes substratos na germinação de sementes de angico (Anadenanthera colubrina (Vell.) Brenan) em condições de laboratório.

Revista Científica Eletrônica de Engenharia Florestal, n.5, 2005.

NAKAGAWA, J. Testes de vigor baseados no desempenho das plântulas. In: KRZYZANOWSKI, F. C.; VIEIRA, R. D.; FRANÇA NETO, J. B.

Vigor de sementes: conceitos e testes. Londrina: ABRATES, 1999. p.2.1-2.24.

PACHECO, M. V. et al. Efeito de temperaturas e substratos na germinação de sementes de Myracrodruon urundeuva Fr. All. (Anacardiaceae). Revista Árvore, v.30, n.3, p.359-367, 2006.

PANTOJA, T. F. et al. Estudo da germinação de Adenanthera pavonina $L$. Disponível em: <http://www.adaltech.com.br/ evento/museugoeldi/resumoshtm/resumos/R09242.htm>. Acesso em: 31 jan. 2006.

POPINIGIS, F. Fisiologia da semente. Brasília: AGIPLAN, 1985. 289p.

PROBERT, R. J. The role of temperature in germination ecophysiology. In: FENNER, M. Seeds: the ecology of regeneration in plant communities. Wallingford: CABI, 1992. p.285-325.
RAMOS, M. B. P.; VARELA, A. P.; MELO, M. F. F. Influência da temperatura e da água sobre a germinação de sementes de paricá (Schizolobium amazonicum Huber ex Ducke - LeguminosaeCaesalpinioideae). Revista Brasileira de Sementes, v.28, n.1, p.163-168, 2006.

ROSA, S. G. T.; FERREIRA, A. G. Germinação de sementes de plantas medicinais lenhosas. Acta Botanica Brasilica, v.15, n.2, p.147-154, 2001.

ROSA, M. F. et al Processo agroindustrial: obtenção de pó de casca de coco verde. Fortaleza: Embrapa Agroindústria Tropical, 2001. 6p. (Comunicado Técnico, 61).

SANTOS, S. R. G.; AGUIAR, I. B. Germinação de sementes de branquilho (Sebastiania commersoniana (Baill.) Smith \& Downs) em função do substrato e do regime de temperatura. Revista Brasileira de Sementes, v.22, n.1, p.120-126, 2000.

VARELA, V. P.; COSTA, A. S.; RAMOS, M. B. P. Influência da temperatura e do substrato na germinação de sementes de itaubarana (Acosmium nitens (Vog.) Yakovlev). Acta Amazonica, v.35, n.1, p.35-39, 2005 b.

VARELA, V. P.; RAMOS, M. B. P.; MELO, M. F. F. Umedecimento do substrato e temperatura na germinação de sementes de angelim-pedra (Dinizia excelsa Ducke). Revista Brasileira de Sementes, v.27, n.2, p.130-135, 2005a.

VAVRINA, C. S. et al. Coconut coir as na alternative to pear meaia for vegetable transplant production. Station Report-Vegetal, v.4, n.sp, p.1-8, 1996.

VÁZQUEZ-YANES, C.; OROZCO-SEGOVIA, A. Patterns of seed longevity and germination in the rainforest. Annual Review of Ecology and Systematics, v.24, p.69-87, 1993. 
